\title{
Myotubular myopathy associated with cataract and electrical myotonia
}

\author{
C. H. HAWKES ${ }^{1}$ AND M. J. ABSOLON \\ From the Wessex Neurological Centre, the General Hospital, Southampton
}

SYNOPSIS A case of myotubular myopathy is described which is unusual because of bilateral cataracts and prominent myotonic-like discharges on the EMG. The significance of these findings is discussed in relation to dystrophia myotonica.

Myotubular myopathy is a slowly progressive muscular disorder starting in childhood, affecting both skeletal and extraocular musculature. Spiro et al. (1966) employed the word 'myotubular' because of the resemblance of the myofibrils to fetal myotubules, and suggested that the disorder might be due to arrested maturation of the developing muscle. This suggestion has been disputed (Sher et al., 1967) and the more neutral term of 'centronuclear myopathy' is preferred by them. There are now more than 20 reports of the disorder but none mentions the presence of cataract and very few have discovered myotonic discharges on the EMG. The present case had both cataracts and electrical myotonia which initially raised the question of dystrophia myotonica until the muscle was examined histologically.

\section{CASE REPORT}

The patient was first seen by us at the age of 29 years, when he was admitted to the Wessex Neurological Centre for assessment of muscular weakness. Hitherto, he had been assumed to suffer from dystrophia myotonica but there were several inconsistent features.

At the age of 2 years he was noted to have a contracture of both Achilles tendons and at 4 years displayed torticollis as a result of contracture of the right sternomastoid muscle. When 10 years old he was found to have bilateral ptosis, severe ophthalmoplegia, diffuse muscular weakness, and wasting involving the face, limb girdles, and limbs, but no

1 Address for reprint requests: Dr C. H. Hawkes, Wessex Neurological Centre, The General Hospital, Southampton SO9 4XY.

(Accepted 25 April 1975.) myotonia on bedside testing. An EMG and muscle biopsy were performed then (1959) but no firm diagnosis could be reached.

On admission to the Wessex Neurological Centre, it was clear that there had been some progression of his weakness especially in the legs. No myotonia could be detected after percussion or voluntary contraction. The neck muscles were affected to a moderate degree but by no means selectively. No cataracts were detected on routine ophthalmoscopy but slit lamp examination revealed curious small midcortical, lens opacities on both sides (Fig. 1). They all had an irregular shape: some were white, but others were blue centrally and white at their edges.

Both parents were examined clinically. The mother was normal but the father had a congenital ptosis of one eye and by ophthalmoscopic examination stippled opacities were found in both lenses probably similar to those of his son.

INVESTIGATIONS The following tests were normal or negative: haemoglobin, white cell count, ESR, liver function tests, serum proteins, porphyrins, serum calcium, phosphate, creatine phosphokinase and cholesterol, glucose tolerance test, protein-bound iodine, blood cortisol, autoimmune profile (including skeletal muscle antibodies), electrocardiogram, and chest radiograph. The skull radiograph showed a very small pituitary fossa at the lower range of normal; there was no thickening of the skull vault. An edrophonium test was negative.

ELECTROMYOGRAM Using a Medelec M6 electromyograph, needle electrode samples were taken from the deltoid, biceps, brachioradialis, quadriceps, and tibialis anterior muscles. Fibrillation potentials were detected in the quadriceps. Striking high frequency discharges were elicited after needle movement or 


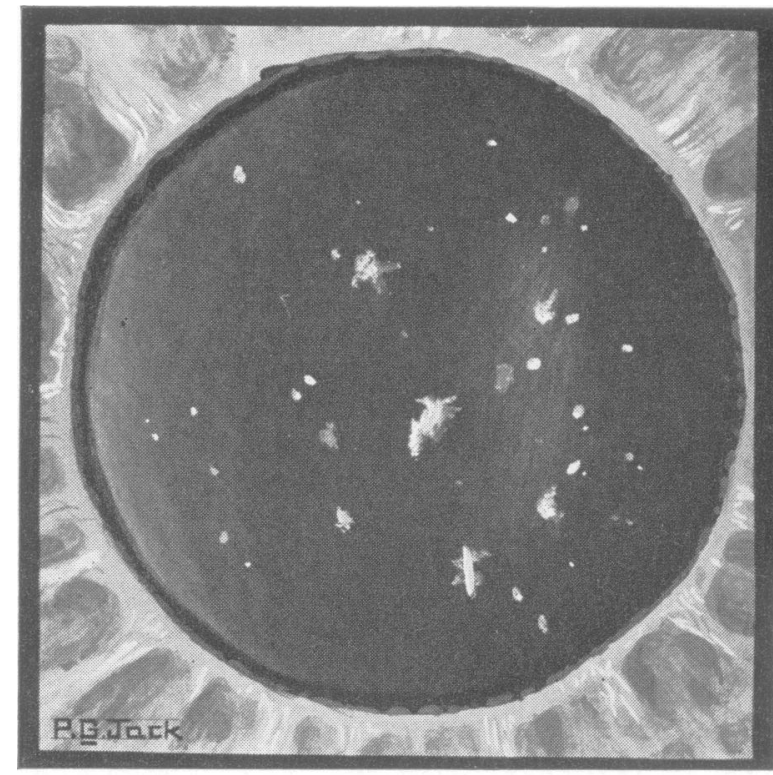

(a)

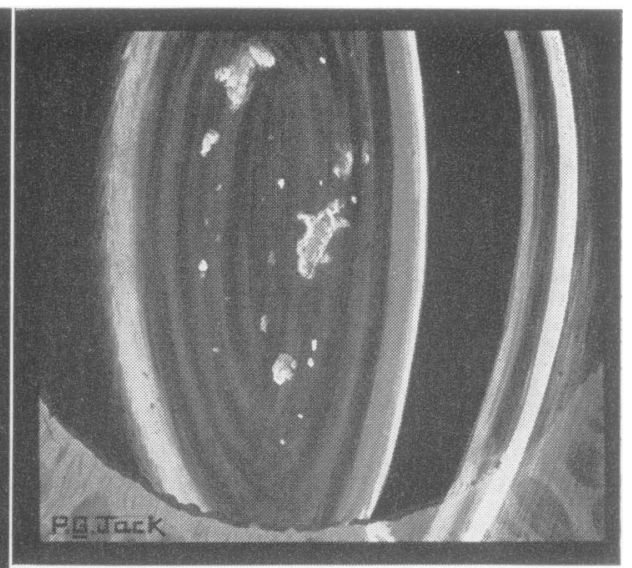

(b)

FIG. 1 Wide and narrow beam views of the lens opacities drawn through slit lamp.

muscle percussion in both forearms and legs. The majority of these were of abrupt onset and termination, and of constant frequency, but several were prolonged (over 60 seconds) with a slow reduction of frequency while maintaining a constant amplitude (Fig. 2). On contraction a highly polyphasic interference pattern of reduced amplitude $(0.5-1.0 \mathrm{mV})$, classical of a myopathy, was obtained in all muscles. An EMG of the ocular muscles revealed myopathic changes but no high frequency discharges. Motor nerve conduction in the median and common peroneal nerves was normal.

MUSCLE BIOPSY A biopsy specimen was obtained from the left deltoid muscle before electromyography. The main histological studies were done on cryostat sections using the following stains: haemotoxylin and eosin (H and E), Sudan, Gomori trichrome, SDH, DPNH, myosin ATP-ase, and phosphorylase.

The cryostat embedded sections revealed two main populations of fibres intimately mixed (Fig. 3). The majority of fibres were small (mean diameter, $30-60 \mu \mathrm{m})$ and all had one or more central nuclei, often in groups or chains. The large fibres (mean diameter $87 \mu \mathrm{m}$ ) also contained one or more nuclei but these were scattered throughout the sarcoplasm. There was no evidence of phagocytosis of muscle fibres, degeneration or regeneration but extensive fatty infiltration was apparent. The blood vessels were normal.

With oxidative stains, the small fibres appeared darker with both SDH and DPNH although the perinuclear area was pale. No differentiation was seen on staining for myosin ATP-ase but the small fibres again stained darker with phosphorylase and PAS stains-likewise showing a perinuclear clear zone. Electron microscopy showed that a small perinuclear clear zone was sarcoplasm containing ribosomes etc.

\section{DISCUSSION}

The clinical and pathological spectrum of myotubular myopathy has been well reviewed in the past (Campbell et al., 1969; Bradley et al., 1970). Despite the variability of its pattern, there is no doubt that our patient fits the general description of the disease.

On clinical examination, the resemblance to dystrophia myotonica was only superficial. While there was striking ptosis, there was no premature baldness and the almost total ophthalmoplegia would be unusual in all but late cases of dystrophia myotonica. Although the neck muscles were involved, they were not 


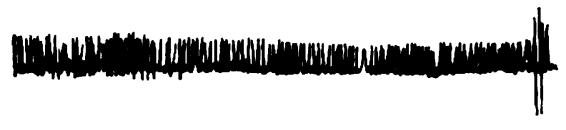

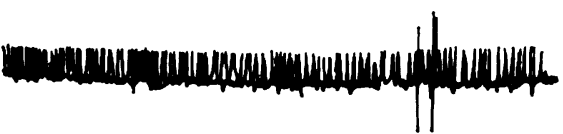

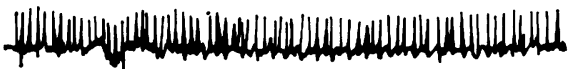
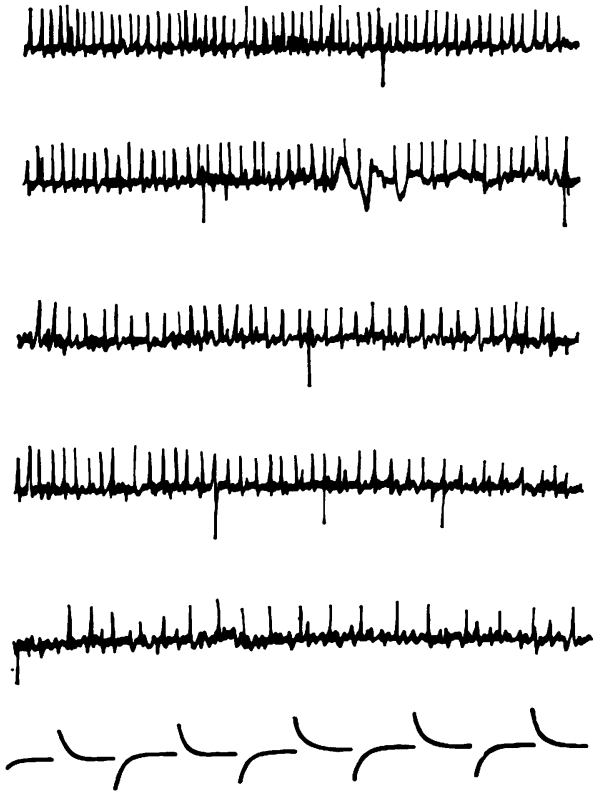

affected in a selective manner. Hitherto, there have been no reports of cataract in myotubular myopathy, whereas in dystrophia myotonica they are a frequent occurrence and may take various forms - both in shape and colour (Walsh, 1957) and indeed may be similar to those noted in the present case (Walsh, 1974-personal communication). It should be emphasized that the lenticular opacities in our patient were seen with the slit lamp and not readily with the ophthalmoscope, hence it may be a feature more common than is generally appreciated.

The EMG was unusual for a myopathy in two respects: (1) the presence of fibrillation (2) high frequency discharges. There have been several reports of the EMG changes in myotubular myopathy but none in great detail. The majority have been compatible with a myopathy (Bradley et al., 1970). Several have detected fibrillations (Spiro et al., 1966; Engel et al., 1968; Munsat et al., 1969; Bethlem et al., 1969, 1970; Winjgaarden et al., 1969), but 'myotonic' discharges are mentioned only by Munsat et al. (1969). Fibrillation occurring in myopathy is unusual but occasionally found in Duchenne type muscular dystrophy and in polymyositis (Lenman and Ritchie, 1970). Apart from the primary myotonic disorders, myotonic-like discharges have

FIG. 2 A myotonic discharge obtained from the tibialis anterior muscle lasting more than 80 seconds. Calibration signal $100 \mu \mathrm{V}, 1 \mathrm{~s}$.

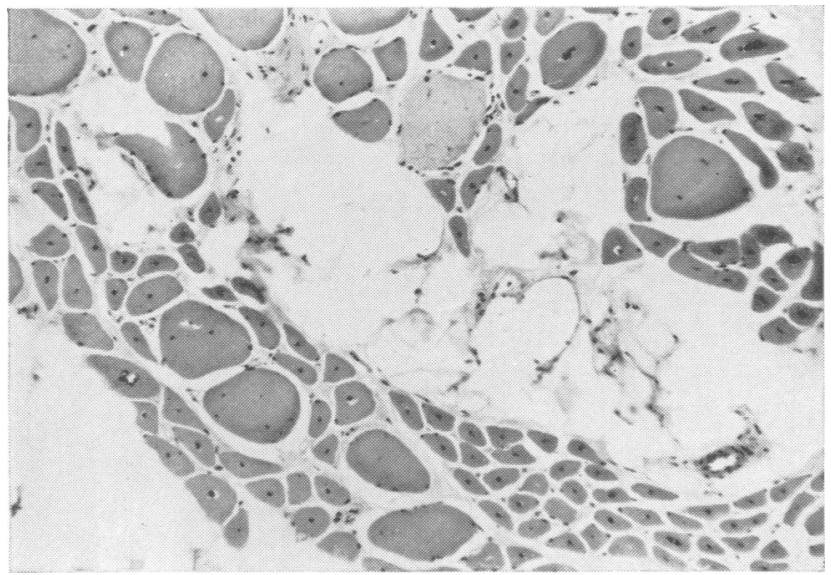

FIG. $3 H$ and $E$ cryostat section obtained from the deltoid muscle. $\times 90$. 
been noticed only in polymositis and neuropathies. Munsat et al. (1969) do not give detail regarding the myotonia in the patient; this is unfortunate as it is important to distinguish true from pseudo-myotonic discharges. According to Buchthal and Rosenfalck (1963), pseudo-myotonic discharges are of high and relatively constant frequency, beginning and ending abruptly, whereas in true myotonia there is a continual increase and decrease in frequency. In the present case, there were many features of pseudomyotonia but some regions showed trains of impulses of decreasing frequency which might be considered to be true myotonia.

The association of myotonia and cataract has indeed been the source of recent interest in view of the induction of both features in experimental animals by diazacholesterol (Peter et al., 1973) and the postulate that there might be a primary disorder of membrane structure causing both myotonia and cataract.

There was little to suggest that the pathological features of our case had much in common with dystrophia myotonica. The appearance of two distinct muscle fibre populations with central nuclei which we observed has never been described in dystrophia myotonica. Furthermore, there was no sign of fibre splitting or ringbinden. However, Campbell et al. (1969) note certain similarities between the histology of dystrophia myotonica and myotubular myopathy, in particular with regard to the appearance of the very large fibres and sacroplasmic masses in dystrophia myotonica. Hence the presence of cataract and myotonic-like discharges in our case might imply that there is a continuous spectrum of change between the two disorders.
We would like to express our thanks to Dr G. S. Graveson for permission to report this case and for his comments on the text. We are also indebted to Dr R. O. Weller for his advice and guidance on the pathological aspects.

\section{REFERENCES}

Bethlem, J., Van Winjgaarden, G. K., Meijer, A. G. F. H., and Hulsman, W. C. (1969). Neuromuscular disease with type 1 fibre atrophy, central nuclei and myotube-like structures. Neurology (Minneap.), 19, 705-710.

Bethlem, J., Van Winjgaarden, G. K., Mumenthaler, M., and Meijer, A. G. F. H. (1970). Centronuclear myopathy with type 1 fibre atrophy and 'myotubules'. Archives of Neuro$\log y$ (Chic.), 23, 70-73.

Bradley, W. G., Price, D. L., and Watanabe, C. K. (1970). Familial centronuclear myopathy. Journal of Neurology, Neurosurgery, and Psychiatry, 33, 687-693.

Buchthal, F., and Rosenfalck, P. (1963). In Muscular Dystrophy in Man and Animals, pp. 208-209. Edited by G. H. Bourne and N. Golarz. Karger: Basle.

Campbell, M. J., Rebeiz, J. J., and Walton, J. N. (1969). Myotubular, centronuclear or pericentronuclear myopathy? Journal of the Neurological Sciences, 8, 425-443.

Engel, W. K., Gold, G. N., and Karpati, G. (1968). Type 1 fibre hypotrophy and central nuclei. Archives of Neurology (Chic.), 18, 435-444.

Lenman, J. A. R., and Ritchie, A. E. (1970). Clinical Electro- $\square \infty$ myography. Pitman Medical: London.

Munsat, T. L., Thompson, L. R., and Coleman, R. F. (1969). Centronuclear ('myotubular') myopathy. Archives of Neurology (Chic.), 20, 120-131.

Peter, J. B., Andiman, R. M., Bowman, R. L. and Nagatomo, T. (1973). Myotonia induced by diazacholesterol; increased $\left(\mathrm{Na}^{+}+\mathrm{K}^{+}\right)$ATP-ase activity of erythrocytic ghosts and development of cataracts. Experimental Neurology, 41, 738-744.

Sher, J. H., Rimalovsky, T. J., Athanassiades, and Aronson, S. M. (1967). Familial centronuclear myopathy. Neurology (Minneap.), 17, 726-742.

Spiro, A. J., Shy, G. M., and Gonotas, N. K. (1966). Myotubular myopathy. Archives of Neurology (Chic.), 14, 1-14.

Walsh, F. B. (1957). Clinical Neuro-ophthalmology, p. 768. Williams and Wilkins: Baltimore.

Winjgaarden van, G. K., Fleury, P., Bethlem, J., and Meijer, A. G. F. H. (1969). Familial 'myotubular' myopathy. Neurology (Minneap.), 19, 901-908. 